\title{
HEALTH-RELATED QUALITY OF LIFE MEASURE BASED ON THE MINIMUM DATA SET
}

\author{
Walter P. Wodchis \\ Toronto Rehabilitation Institute and Institute for Clinical Evaluative Sciences \\ John P. Hirdes \\ University of Waterloo and Homewood Research Institute \\ David H. Feeny \\ University of Alberta
}

\begin{abstract}
Objectives: To introduce a health-related quality of life measure for home care and institutional longterm care settings based on the Minimum Data Set (MDS) and the Health Utilities Index Mark 2 (HUI2). Methods: Health attributes of Health Related Quality of Life (HRQOL) were identified, and suitable constructs were determined. Items from the MDS were mapped to the HUI2. Scores for the Minimum Data Set Health Status Index (MDS-HSI) were calculated using the HUI2 scoring function. Measurement properties are examined and reported. HRQOL scores were compared across study populations and to an external reference population. Random samples were drawn from long-term care clients in private households $(n=377)$, supportive housing apartments $(n=80)$, two residential care facilities $(n=166)$, and a chronic care hospital $(n=274)$ in Ontario, Canada. All sampled residents were assessed for health-related items using the MDS.

Results: The MDS-HSI results provide preliminary evidence of good validity. Institutional populations had lower overall HRQOL scores than community populations. Comparisons to existing Canadian national data support construct validity.

Conclusions: The MDS-HSI provides a summary outcome measure and an indicator of health status in the six supporting attributes. Longitudinal research is required to assess the sensitivity of the measure to changes over time. Further research is also required to establish the consistency between the preference weights used in this application of the $\mathrm{HUI} 2$ and those that would be derived from a frail elderly population.
\end{abstract}

Keywords: Health-related quality of life, Outcomes, Long-term care, Minimum data set, Health utilities index

Health-related quality of life (HRQOL) is considered an important outcome for geriatric care $(1 ; 4 ; 6 ; 20 ; 47)$. The development of HRQOL instruments is a major interest for clinical trials and technology assessment $(38 ; 46)$. Including HRQOL assessment in clinical and health services research is useful, because it can allow consistent comparison of residents with

This research was supported by the Seniors Independence Program (grant 4687-06-93-458) and the Providence Centre Foundation. Thanks to Copernicus Lodge, Providence Centre, and the Red Cross Homemaker Program for participating in this study. Also thanks to Roger Roberge and Dan Dekoker from Statistics Canada for their help in obtaining and analyzing the NPHS data. International copyright for the MDS-NH and MDS-HC is held by interRAI. International copyright for HUI2 and HUI3 materials are held by Health Utilities, Inc. 
different health impairments. A standardized HRQOL measure can be used to discriminate among respondents at a point in time, predict future outcomes, measure changes over time, and to evaluate the appropriateness and effectiveness of individual and system outcomes and the means by which these are achieved (32). At the same time, measuring summary HRQOL for elderly individuals with chronic or long-term conditions is difficult, because patients face different goals and outcomes depending on their physical health and psycho-social and well-being status.

Health status assessment in the elderly traditionally uses scales that assess physical or cognitive performance and few measures have been used to assess overall health-related outcomes for this population. Summary HRQOL measures are typically developed and tested in more general population groups. The present research applies knowledge that was generated in the HRQOL field to research involving the elderly population.

HRQOL measurement has been defined as "the value assigned to duration of life as modified by the impairments, functional states, perceptions, and social opportunities that are influenced by disease, injury, treatment, or policy" (33). Existing HRQOL measures include attributes such as medical symptoms and side effects $(3 ; 11 ; 25 ; 42)$; functional ability in activities such as mobility $(3 ; 11 ; 25)$ and self-care $(3 ; 11 ; 42)$ (e.g., toileting, eating); psychological elements, including cognitive and emotional functioning $(3 ; 11 ; 42)$; and sensory abilities such as vision, hearing, and speech $(25 ; 42)$. An HRQOL measure should provide both a single summary outcome measure and scores for several subscales that represent health status in specific HRQOL attributes.

Despite the potential usefulness of HRQOL measurement, there are still relatively few applications in the elderly population. An HRQOL measure for disabled elderly populations who receive long-term care services would be helpful; a measure that could be available for a wide variety of elderly populations would be particularly useful for evaluators and clinical researchers. The purpose of this study is to introduce an HRQOL measure for use in community and institutional long-term care settings. The measure introduced in this study is derived by mapping items from the Minimum Data Set (MDS) series of instruments into the Health Utilities Index Mark 2 (HUI2) to obtain an overall HRQOL score and six attribute-specific summary scores of HRQOL.

The MDS is the most widespread health assessment instrument for geriatric settings (23). It is used in over twenty countries, including mandated use in all United States' nursing homes (44) and long-term care facilities in four Canadian provinces. Several parallel instruments have been developed for, home care (MDS-HC), mental health (MDS-MH), acute care (MDS-AC), and postacute care (MDS-PAC). The MDS-HC is being implemented by nine U.S. states and six Canadian provinces. These instruments consist of a core set of common assessment items and are supplemented by items that are particular to their respective settings (e.g., the MDS-HC includes instrumental activities of daily living such as meal preparation). Extensive evaluation has been done to provide evidence of the reliability and validity of these instruments and their subscales $(12 ; 28 ; 48)$. In particular, the Cognitive Performance Scale (CPS) is computed from MDS variables to determine the cognitive ability of residents on a scale that ranges from a score of 0 (cognitively intact) to a score of 6 (severely impaired). The associated MDS items, including comatose, impaired daily decision making, ability to make oneself understood, short-term memory, and dependent eating, all have reliability Kappas greater than 0.9 (29). A hierarchical measure of selfperformance in activities of daily living (ADL) is based on MDS items for personal hygiene, locomotion, eating, and toileting (30;48). Applications of the MDS instruments include care planning (36), case-mix (13), quality improvement (21), and outcome measurement $(22 ; 23)$. To date, a single summary measure of HRQOL has not been available for the MDS.

Development of an MDS-based HRQOL measure would facilitate program evaluation and outcome studies for long-term care programs as well as clinical trials and technology 
assessment involving this population. An appropriate HRQOL measure should satisfy several criteria. First, it should identify distinct attributes of HRQOL applicable to a frail elderly population, including ADLs and cognition. Second, it should provide a score representing health status in each health attribute, and a single summary score of HRQOL. Third, the summary score should have interval-scale properties to facilitate powerful and simpler analyses than would be possible with numerous ordinal or categorical outcomes. Additionally, an index that integrates morbidity and mortality would allow quality-adjusted life-expectancy values to be calculated. This may be particularly useful in the frail elderly population where multiple chronic conditions and mortality are both substantial concerns in evaluating outcomes.

\section{CONCEPTUAL MODEL}

A standard approach to developing an HRQOL measure begins by establishing a conceptual framework of HRQOL and contributing attributes. Items to measure the conceptual model are identified and scales are constructed from included item scores. Instead of developing a new conceptual model, we examined existing HRQOL measures to identify an existing model suitable for the elderly population. We evaluated existing measures with the goal of ensuring that attributes most important for the elderly population (e.g., ADL, cognition) were included. After selecting an existing measure, an algorithm was created that links MDS health status items to the existing measure.

Five widely used generic HRQOL measures that have demonstrated reliability and validity in several settings are the SF-36 (45), the EuroQol (EQ-5D) (11), the Quality of Well Being (QWB) (25), the Sickness Impact Profile (SIP) (3), and the HUI2 (42). The SF-36 includes profile scores for physical and mental health attributes but does not include a self-care attribute. All SF-36 scores are measured with ordinal scale properties, and there is no explicit relationship between the seven available profile scores and an overall health status score. There are, however, summary physical and mental health scores. The EQ5D includes mobility, anxiety/depression, usual activities, pain, and self-care but does not assess cognition or sensation attributes. The QWB includes mobility, physical activities, social activities, and a symptom problem complex. The SIP only provides scores that reflect morbidity and, therefore, cannot integrate mortality and morbidity. The HUI 2 system was adopted for our conceptual model because it provides a comprehensive summary of health status and provides interval scale scores for six distinct attributes, including both pain and self-care, which are particularly relevant for chronically ill elderly patients. The HUI2 scoring function is used to assign utility or preference scores associated with various health states. The HUI2 scoring function has been used to provide provisional scores for the Ontario Health Survey (5;43), the Statistics Canada 1991 General Social Survey (43), the Statistics Canada 1994 and 1996 National Population and Health Survey (NPHS) (39). The HUI2 score function has also been used in a study of patient and proxy HRQOL for Alzheimer's patients (31). We compare the results from the present study with the aforementioned NPHS results.

All summary interval-scale HRQOL scores are based on a weighted function of individual health status items. Whereas some HRQOL scales use statistical methods to develop the required weights, others create a summated score by summing together scores from related questionnaire items. This approach suggests an equal weight for all constituent HRQOL attributes. In contrast, the HUI2 weights are based on expressed population preferences among various possible health states. One concern is whether the existing HUI2 weights are appropriate for use with the elderly population. The HUI2 preference scoring function embodies the preferences of "person mean" from a representative sample. Some potential problems may exist with this approach. It may be the case that the preferences of the general 
population do not fully match those of particular groups. Nonetheless, existing evidence indicates that, although there is considerable heterogeneity in preference scores among individuals, little of the observed heterogeneity is systematically related to sociodemographic characteristics such as age (7), gender (37), income (15), education, religion, or country of residence $(10 ; 34)$. Although a few studies have detected some effects due to age or gender $(9 ; 40)$, in general, there is little evidence of systematic effects $(14 ; 16 ; 17)$. The evidence to date suggests that at the "mean" level, preferences obtained from representative samples generalize well $(2 ; 24)$. Furthermore, the weights from the HUI2 have been used in studies of geriatric population outcomes $(31 ; 39 ; 43)$.

This study presents the results of combining MDS health assessment information with HUI2 weights to derive an HRQOL measure: the Minimum Data Set Health Status Index (MDS-HSI) for community and institutional long-term care settings. The purpose of this study is to present the development of the MDS-HSI and provide preliminary results assessing the validity of the new measure.

\section{METHODS}

\section{Minimum Data Set}

This study uses the MDS for nursing homes v2.0 (MDS-NH) and the MDS for home care (MDS-HC) assessment instruments to assess health status in long-term care. The MDS-HC assesses over 200 functional, health status, social environment, and service items; 114 of these are common to the MDS-NH. The MDS-NH is used for institutional settings and includes detailed information about nursing activities, whereas the MDS-HC is used for home care and supportive housing populations and includes detailed information about informal care activities. The average weighted Kappa inter-rater reliability level for the MDS-NH is 0.79 , indicating excellent reliability (19). The reliability values for extra items on the MDS-HC are similarly high, with the weighted Kappa averaging 0.70 (28). The average reliability for items included in the present analysis is 0.83 .

Sgadari et al. (41) showed that the MDS demonstrated high reliability in virtually all attributes in a cross-national comparison involving eight countries. Studies have shown the MDS CPS to be highly correlated with the Folstein Mini-Mental State Exam (MMSE), a widely used screening tool for cognition (18). Similarly, the MDS ADL Hierarchy has been validated against the Functional Independence Measure (FIM) (30). Like any instrument, the MDS can yield poor quality data if not implemented appropriately or if used by untrained personnel (see, for example, Crooks et al. study of continence ratings) (8). However, Phillips and Morris (35) demonstrated that MDS data from administrative records generally yield findings comparable to those with data obtained by trained research nurses. Therefore, MDS instruments are suitable for clinical evaluations of health and functional ability in older populations.

\section{Health Utilities Index}

The HUI2 multi-attribute health status classification system and associated preference weights are used to facilitate the development of a single summary measure of HRQOL using MDS data. The re-assessment of preferences associated with health states was beyond the scope of the current study; therefore, the community HUI2 preference weights are used here.

The MDS assessment data provide reliable measures of health states, which are then matched to the HUI2 classification system. The latter originally included seven attributes to define health states: sensation (vision, hearing, speech), mobility, emotion, cognition, selfcare, pain, and fertility. Analogous to other studies using the HUI2 in population surveys 
$(39 ; 43)$, we use six attributes in this study; the seventh attribute, fertility, was not assessed in this study. For purposes of scoring, we have used the same approach as used in the Canadian NPHS (our comparison population) by assigning level 1 (normal for their age) fertility for all subjects. All individuals in the current study, hence, are presumed to have normal fertility in so far as it affects their overall HRQOL. In contrast to survey questionnaires for developed health indices (including HUI2), MDS assessments are not solely based on resident selfreported health status. Assessment information is compiled by nurses who use all available sources of information, including observed function and medical charts as well as resident and family report. Relying solely on self-report information is problematic for the present application, because it is not available for elderly individuals with dementia.

Two versions of the HUI are in common use: the Mark 2 system (HUI2) and the Mark 3 system (HUI3). Although there is overlap between several of the attributes of health status in the two systems, each is a comprehensive stand-alone system in its own right. The HUI2 has been adopted in this study for several reasons. The HUI2 provides a direct assessment of self-care, which is thought to be highly relevant in the context of long-term care. The concepts of pain differ in the two systems. Although both assess the degree of severity, HUI2 considers the analgesic required for pain control, whereas HUI3 focuses on the degree of disruption in normal activities. In the HUI2, emotion refers to worry and anxiety, whereas the HUI3 focuses on happiness versus depression. The HUI2 groups vision, hearing, and speech together; these are broken down into their constituent parts in the HUI3. We have adopted the HUI2 for our study of long-term care settings because (1) it focuses on self-care, (2) the HUI2 pain attribute considers pain in the context of treatment patterns; (3) worry and anxiety are more readily observable (by MDS assessors) than the construct of happiness versus depression. Nonetheless, future development of a HUI3 crosswalk may also be of value.

\section{Mapping}

Several steps were used to map items from the MDS assessment to the HUI2 attribute definitions. First, each of the six attributes was linked with all related items in the MDS-NH and MDS-HC. Table 1 provides a list of health attributes in the MDS assessments and the HUI2 classification system. Each category in the MDS consists of several questions/items. Relevant items for each attribute were identified from the MDS and a mapping algorithm was developed to associate levels on MDS items with the attribute definitions (levels)

Table 1. Attributes of Health: MDS and HUI2 ${ }^{\mathrm{a}}$

\begin{tabular}{ll}
\hline MDS-NH/home care & \multicolumn{1}{c}{ HUI2 } \\
\hline $\begin{array}{l}\text { Cognitive patterns } \\
\text { Communication/hearing patterns/vision }\end{array}$ & $\begin{array}{l}\text { Cognition } \\
\text { Sensation (vision, hearing, speech) } \\
\text { Eood and behavior patterns }\end{array}$ \\
$\begin{array}{l}\text { Social functioning } \\
\text { Physical functioning }\end{array}$ & Mobility, self-care \\
$\begin{array}{l}\text { Continence } \\
\text { Disease diagnoses }\end{array}$ & \\
$\begin{array}{l}\text { Health conditions (including pain) } \\
\text { Nutrition/hydration status }\end{array}$ & Pain \\
$\begin{array}{l}\text { Dental status } \\
\text { Skin condition }\end{array}$ & \\
$\begin{array}{l}\text { Service utilization } \\
\text { Medications }\end{array}$ & \\
& \\
\end{tabular}

${ }^{a}$ MDS, Minimum Data Set; HUI2, Health Utilities Index Mark 2; NH, nursing home. 
in the HUI2 classification system. We used the MDS CPS (18) to map to the definitions in the HUI2 cognition attribute, because it is a validated scale that already provides categories that are consistent with the HUI2 definitions. Table 2 describes the mapping algorithm used to map MDS items into HUI2 attribute definitions. The far right column of Table 2 also provides the multi-attribute preference weights associated with each attribute level. The scores are reproduced from Torrance et al. (42).

There are a few differences in items available from the MDS-NH and the MDS-HC instruments used in the mapping to HUI2 definitions. A total of thirty-four items were selected for the mapping algorithm. Of the items selected for the mapping, twenty-three were identical on both instruments, eight assessed the same indicator but were based on slightly different scales, two items appeared only on the MDS-HC, and three items appeared only on the MDS-NH. Specifically, the use of equipment used for mobility is measured slightly differently on the MDS- HC and the MDS-NH. On the MDS-HC, four separate items are used to indicate what equipment is used (e.g., cane, walker, wheelchair), whereas the MDS-NH uses two items to identify the most common equipment used. There is no anticipated impact from these differences, because the mapping algorithm for HUI2 mobility attribute only requires knowing whether any equipment is used. Even though a comatose measure contributes to the CPS, it was not included in the MDS-HC. The expected rarity of finding comatose individuals in community dwellings justified its exclusion from this version of the MDS. There is no anticipated impact from the comatose indicator not being present in the MDS-HC, because the two highest levels of the CPS are considered equivalent to the highest level of cognitive impairment in the HUI2. Only the MDS-NH includes items for the use of hearing and vision aids (both MDS instruments include assessments of ability in the presence of aids if used). We examined the potential impact of this missing data by assessing the number of individuals assessed with the MDS-NH who showed no limitations but did use these aids. The final difference relates to the measurement of pain. In this case, the MDS-HC provides a complete assessment of relevant components (frequency, intensity, activity disruption, and relief from medications), whereas the MDS-NH does not assess the latter two indicators in relation to pain. The approach used by Torrance et al. (43) for mapping from the Ontario Health Survey to HUI2 was used to compensate for the missing data. It was assumed that individuals experiencing severe and daily pain experience disruption in their daily activities. The presence of severe and daily pain, hence, is assigned to a level of 4.5 in the pain attribute ( 5 levels). An interpolation between the utility scores for level 4 and level 5 (the highest pain level) provides an approximation of the score for the mid-point. This strategy is justified under the linearity of the single-attribute preference scoring function. In the application of the mapping, sensitivity analysis was used to determine the impact of these approximations.

\section{Scoring}

Scores assigned to each health state depicted in the HUI2 embody the preferences of a representative sample from the community. Preference scores for the HUI2 system were obtained using a standard gamble (SG) methodology in a study carried out at McMaster University. The SG approach is recommended as the most suitable approach for developing health state utility scores $(26 ; 42)$. In the original study, the SG process was preceded by a ranking of health conditions using a visual analog scale (42). A multiplicative multi-attribute utility function based on SG scores is used in this study.

Health status, described in the HUI2 as six attribute-vectors, is valued using a multiplicative preference scoring function (based on Torrance et al.) (42) defined as:

$$
\mathrm{HSI}_{\mathrm{i}}=\left(\alpha * \prod_{\mathrm{k}=1}^{6} \mathrm{w}_{\mathrm{k}}\left(\mathrm{h}_{\mathrm{i}, \mathrm{k}}\right)\right)-\beta
$$


Table 2. MDS-HUI2 Multi-attribute Health Status Index Mapping

Attribute

MDS variable

HUI2 level, description, and preference

weight $^{\mathrm{a}}$

Sensation No vision, hearing, or communication difficulty

(vision, Minimal impairment (with or without aids)

hearing, Highly impaired (with or without aids)

speech)

Severely impaired (with or without aids)

Mobility Independent in locomotion

Supervision but no physical help from others \& no devices

No physical help \& self-supporting devices (cane, walker, self-wheel)

Physical help from others \& use of devices

Total dependence on others

Emotion No negative mood indication in last 30 days (5 indicators)

Up to two indications exhibited 1-5 days per week

Any one daily or at least three exhibited 1 to 5 days per week

Two or three indicators exhibited daily

Four or five indicators exhibited daily

Cognition No problem with memory or decision-making

Memory problem or mild impairment in decisionmaking

Moderate impairment in decision-making

Severe impairment in decision-making

Self-care Independence in all these activities

Supervision by others but no assistance for any of these activities

Limited assistance in any of these activities

Extensive assistance or total dependence in any of these activities

Pain No pain

Pain less than daily, not requiring prescribed medications

Pain daily, not intense, disrupts activities, and relieved by medication

Pain daily, intense, disrupts activities, and relieved by medication

Pain daily, intense, disrupts activities, and not relieved by medication
1. Able to see, hear, and speak normally for age

2. Requires equipment to see or hear or speak

3. Sees, hears, or speaks with limitations, even with 0.86 equipment

4. Blind, deaf, or mute

1. Able to walk, bend, lift, jump and run normally for age

2. Walks, bends, lifts, or jumps with some

limitations; no help required

3. Requires mechanical equipment (such as canes, crutches, braces or wheelchair) to walk or get around independently

4. Requires the help of another person to walk or get around and requires equipment

5. Unable to control or use arms and legs

1. Generally happy and free from worry

2. Occasionally fretful, angry, irritable, anxious, depressed, or suffering "night terrors"

3. Often fretful, angry, irritable, anxious, or depressed

4. Almost always fretful, angry, irritable, anxious, or depressed

5. Extremely fretful, angry, irritable, anxious, or depressed usually requiring hospital or psychiatric care

1. Learns and remembers normally for age

2. Learns and remembers more slowly than peers

3. Learns and remembers very slowly

4. Unable to learn and remember

1. Eats, bathes, dresses, and uses toilet normally for age

2. Eats, bathes, dresses, or uses the toilet independently with difficulty

3. Requires equipment to eat, bathe, dress, or use the toilet independently

4. Requires help of another person to eat, bathe, dress, or use the toilet

1. Free from pain and discomfort

2. Occasional pain. Discomfort relieved by nonprescription drugs or self control without activity disruption

3. Frequent pain. Discomfort relieved by oral medicines with occasional disruption of normal activities

4. Frequent pain; frequent disruption of normal activities. Discomfort requires prescription narcotics for relief

5. Severe pain; pain not relieved by drugs and constantly disrupts normal activities

a Source: Torrance et al. (42).

MDS, Minimum Data Set; HUI2, Health Utilities Index Mark 2. 
where: $\alpha$ and $\beta$ are parameters providing the range for the index scores.

$$
\begin{aligned}
\alpha= & 1.06 \text { and } \beta=0.06 \\
\mathrm{w}_{\mathrm{k}}\left(\mathrm{h}_{\mathrm{i}, \mathrm{k}}\right)= & \text { the preference weight or value associated with health state } \mathrm{h}_{\mathrm{i}, \mathrm{k}} \text { for } \\
& \text { individual } \mathrm{i} \text { on attribute } \mathrm{k} .
\end{aligned}
$$

From the attributes and the HUI2 multi-attribute preference scores shown in Table 2, the MDS-HSI is calculated for each health state. For example, an individual at the third level of the sensation attribute (sees, hears, or speaks with limitations, even with equipment) and full functionality for all other attributes would be assigned an HRQOL score of 0.85 $(1.06 *(0.86 * 1 * 1 * 1 * 1 * 1))-0.06)$. The score derived as the MDS-HSI represents the preference score of a particular health state. To justify the summation over individuals, we assume that the same health states are of equal value to all individuals. Using these weights and parameters results in perfect health having a score of 1.00 , whereas dead is represented by 0.0 . The worst of all HUI 2 health states has a negative preference score of -0.03 as it was judged by respondents to be worse than dead. The standard error of the scoring function is 0.015 (0.06 including the model error) (42).

\section{Validity}

It should be recognized that establishing validity is an ongoing process involving the accumulation of evidence for different types of validity (27). In the present research, we use several approaches to provide preliminary evidence for the validity of the MDS-HSI. Adopting the HUI2 as the standard of HRQOL, we determined content or face validity by the correspondence between MDS items and HUI2 definitions as depicted in Table 2. Assessment of criterion validity is more difficult because there is no existing "gold-standard" for overall HRQOL or most of the health attribute scores. We examine the correspondence of the MDS-HSI with existing instruments for two attributes where we do have validated MDS-based gold-standard measures (CPS for cognition and ADL hierarchy for self-care). In addition, we examine the correlation of MDS-HSI scores with summated scores from MDS items associated with each health attribute. Summated scores were constructed by adding together scores for all MDS items that were used in the mapping from MDS to HUI2. These scores are not part of the original MDS instrument and are only created to provide an additional comparison for the MDS-HSI scale. Summated scores are a convenient and common approach to creating scales. Although summated scores do not provide intervalscale scores, the correlation between MDS-HSI scores and the summated scores provides evidence of validity in relation to a commonly used approach to scale construction.

We use two approaches to evaluate construct and convergent validity. First, we compare the MDS-HSI single-attribute scores with summated scales based on the same health attributes, and second, we use HUI2 scores for an external reference population (NPHS) to examine the correspondence of MDS-HSI scores with those obtained for similar populations. Summated scores are calculated as the sum of items included in each health attribute for the MDS-HSI mapping algorithm. Our approach provides useful comparison because health outcome scales are commonly based on the summation of items. Finally, we examine the construct and discriminant validity in reference to hypotheses about MDS-HSI scores in several different health care settings.

\section{Hypotheses}

In this study, several a priori hypotheses about results arising from the MDS-HSI are specified and evaluated to assess the validity of this instrument. A high level of correlation between MDS-HSI attribute scores and gold-standard or summated scores will provide evidence for construct and convergent validity. Hypotheses for patterns across settings are 
used to assess discriminant validity. First, we would expect that average MDS-HSI scores would be highest among the independent community dwelling population and lowest for the institutionalized population. Most individual attribute scores should follow a similar pattern. We expect that problems with self-care, mobility, and cognition would be more apparent in institutional elderly. On the other hand, because we are considering an identical age cohort for all settings, we have no a priori hypotheses that impairments in sensation (vision, hearing, speech) should differ notably across populations. Similarly, pain and emotion scores could be lower (worse) for institutional residents because of greater health impairments or higher (better) because of more intensive treatment.

\section{Study Population}

The health status of long-term care residents in four types of residential settings was assessed using the MDS assessment instruments. The study participants included 80 residents in a supportive housing setting ( $\mathrm{SH}), 377$ recipients of home care services in the community (HC), 166 residents in two residential facilities (RF), and 274 patients in a chronic care hospital $(\mathrm{CCH})$. Hence, the total sample was 897 individuals from five organizations. All residents were random samples of their respective settings with the exception of the chronic care hospital patients. It is a legislated requirement for all chronic care hospital patients in Ontario to be assessed using the MDS, so full census data are reported for this setting. Data were missing for twenty-four of the home care clients, limiting this population to 353 residents. Although the samples may be considered to be representative of the participating organizations, the five organizations cannot be expected to be fully representative of their respective health care sectors. As data from state/provincial implementations of the MDS$\mathrm{NH}$ and MDS-HC become available to researchers, it will be possible to repeat these analyses with sector-wide comparisons.

Care in each study setting includes personal assistance to residents in ADL function such as bathing and dressing. Nursing services such as medication oversight are also common to all settings. Home care services are delivered to individuals living in independent community dwellings. Supportive housing is similarly an independent dwelling, not a health care facility, and admission is determined in large part by residents' willingness to pay. Supportive housing residents have access to the same care as home care residents, but services are typically provided by on-site staff. In contrast to the home care clients, not all residents in supportive housing use or require health care. Admission to a residential facility is restricted to persons requiring twenty-four-hour supervision. These facilities are required to have nursing staff present twenty-four hours each day. Chronic care hospitals have larger budgets for medical devices, higher staff to resident ratios, and are intended for more medically complex care. The mean age and gender (percent female) for the four study populations are as follows: supportive housing, 82.4 (74\%); home care, 77.8 (78\%); residential facility, $83.2(75 \%)$; chronic care hospital, $74.6(65 \%)$.

Comparisons of MDS-HSI scores from the study population are made to similar populations from the 1994 Statistics Canada NPHS. Because these two measures are similarly constructed and based on the HUI2 multi-attribute classification system, we use the comparison to assess convergent validity (27). ${ }^{1}$ The Statistics Canada 1994 NPHS is a nationally representative sample of residents in the community and residents in institutions $(n=19,600)$. Results were calculated from NPHS data files for each of the two populations. The NPHS data are available through the Statistics Canada Data Liberation Initiative.

The NPHS community populations could include residents from both community (home care) and supportive housing settings, whereas the institutional population includes both residential facility and chronic care hospital residents. Exact age is not available from NPHS survey data but community and institutional populations, respectively, had $20 \%$ and 
$69 \%$ eighty years of age and older. The respective gender compositions were $61 \%$ and $72 \%$ female.

\section{RESULTS}

The resulting distributions for each attribute level in each of the four settings are presented in Table 3. Generally, the distributions across attributes show that residents of more intensive care settings experience higher levels of impairment. The prevalence of full functionality (level $=1$ ) within any attribute typically declines as the care setting becomes more intense.

Results for each population support the study hypotheses. Predominantly, the home care study population experiences moderate levels of functional ability. Seventy-eight $\%$ of this population can walk independently with the aid of assistive devices, but a quarter are fully independent in performing the activities of daily living associated with the self-care attribute. None of the $\mathrm{HC}$ clients are categorized in the highest level of cognitive impairment. Although a third require the use of assistive devices for mobility, approximately $75 \%$ are able to care for themselves. As expected, the residential facilities have a more dependent population

Table 3. Prevalence within Attributes

\begin{tabular}{|c|c|c|c|c|c|c|c|}
\hline Attribute & Level & $\begin{array}{l}\text { Supportive } \\
\text { housing } \\
(n=80)\end{array}$ & $\begin{array}{l}\text { Home care } \\
(n=353)\end{array}$ & $\begin{array}{l}\text { Resident } \\
\text { facility } \\
(n=166)\end{array}$ & $\begin{array}{l}\text { Chronic } \\
\text { hospital } \\
(n=274)\end{array}$ & $\begin{array}{c}\text { NPHS } \\
\text { community } \\
(n=3002)\end{array}$ & $\begin{array}{c}\text { NPHS } \\
\text { institution } \\
(n=1467)\end{array}$ \\
\hline \multirow{4}{*}{$\begin{array}{l}\text { Sensation } \\
\text { (vision, } \\
\text { hearing, } \\
\text { speech) }\end{array}$} & 1 & $45 \%$ & $47 \%$ & $8 \%$ & $27 \%$ & $12 \%$ & $13 \%$ \\
\hline & 2 & $39 \%$ & $37 \%$ & $33 \%$ & $31 \%$ & $74 \%$ & $36 \%$ \\
\hline & 3 & $13 \%$ & $11 \%$ & $33 \%$ & $22 \%$ & $9 \%$ & $44 \%$ \\
\hline & 4 & $3 \%$ & $5 \%$ & $26 \%$ & $20 \%$ & $5 \%$ & $7 \%$ \\
\hline \multirow[t]{5}{*}{ Mobility } & 1 & $65 \%$ & $22 \%$ & $15 \%$ & $34 \%$ & $84 \%$ & $31 \%$ \\
\hline & 2 & $0 \%$ & $0 \%$ & $4 \%$ & $1 \%$ & $3 \%$ & $1 \%$ \\
\hline & 3 & $35 \%$ & $73 \%$ & $44 \%$ & $10 \%$ & $9 \%$ & $30 \%$ \\
\hline & 4 & $0 \%$ & $3 \%$ & $10 \%$ & $15 \%$ & $4 \%$ & $2 \%$ \\
\hline & 5 & $0 \%$ & $2 \%$ & $28 \%$ & $39 \%$ & $0 \%$ & $36 \%$ \\
\hline \multirow[t]{5}{*}{ Emotion } & 1 & $84 \%$ & $53 \%$ & $29 \%$ & $45 \%$ & $75 \%$ & $28 \%$ \\
\hline & 2 & $11 \%$ & $35 \%$ & $30 \%$ & $24 \%$ & $21 \%$ & $38 \%$ \\
\hline & 3 & $4 \%$ & $10 \%$ & $32 \%$ & $19 \%$ & $3 \%$ & $30 \%$ \\
\hline & 4 & $1 \%$ & $2 \%$ & $8 \%$ & $12 \%$ & $1 \%$ & $0 \%$ \\
\hline & 5 & $0 \%$ & $0 \%$ & $0 \%$ & $0 \%$ & $0 \%$ & $4 \%$ \\
\hline \multirow[t]{4}{*}{ Cognition } & 1 & $84 \%$ & $65 \%$ & $21 \%$ & $22 \%$ & $62 \%$ & $23 \%$ \\
\hline & 2 & $10 \%$ & $27 \%$ & $22 \%$ & $20 \%$ & $3 \%$ & $27 \%$ \\
\hline & 3 & $6 \%$ & $4 \%$ & $23 \%$ & $25 \%$ & $22 \%$ & $16 \%$ \\
\hline & 4 & $0 \%$ & $4 \%$ & $34 \%$ & $33 \%$ & $13 \%$ & $34 \%$ \\
\hline \multirow[t]{4}{*}{ Self-care } & 1 & $74 \%$ & $26 \%$ & $7 \%$ & $4 \%$ & $97 \%$ & $80 \%$ \\
\hline & 2 & $10 \%$ & $12 \%$ & $8 \%$ & $5 \%$ & $2 \%$ & $2 \%$ \\
\hline & 3 & $15 \%$ & $49 \%$ & $27 \%$ & $19 \%$ & $1 \%$ & $0 \%$ \\
\hline & 4 & $1 \%$ & $13 \%$ & $58 \%$ & $72 \%$ & $0 \%$ & $18 \%$ \\
\hline \multirow[t]{6}{*}{ Pain } & 1 & $46 \%$ & $30 \%$ & $40 \%$ & $45 \%$ & $71 \%$ & $66 \%$ \\
\hline & 2 & $26 \%$ & $20 \%$ & $14 \%$ & $9 \%$ & $6 \%$ & $7 \%$ \\
\hline & 3 & $23 \%$ & $21 \%$ & $16 \%$ & $15 \%$ & $16 \%$ & $14 \%$ \\
\hline & 4 & $2 \%$ & $23 \%$ & n.a. & n.a. & $\mathrm{a}$ & a \\
\hline & 4.5 & n.a. & n.a. & $30 \%$ & $31 \%$ & $7 \%$ & $13 \%$ \\
\hline & 5 & $4 \%$ & $6 \%$ & n.a. & n.a. & $\mathrm{a}$ & a \\
\hline
\end{tabular}

${ }^{\text {a }}$ Levels 4 and 5 are mapped directly from the MDS-HC, while interpolation between the two was used for NPHS and MDS-NH mapping algorithms (see text.)

NPHS, National Population and Health Survey; n.a., not applicable. 
than seen in community settings. A sharp difference in cognitive abilities is particularly evident. Patients in the $\mathrm{CCH}$ had the lowest levels of ability in the self-care attribute, with $72 \%$ requiring regular physical assistance in performing these activities. More than half of the patients in this facility had negative mood patterns. This observation is consistent with other studies on the morbidity burden experienced by residents of institutional care.

NPHS results were calculated for survey respondents over the age of sixty-four, ensuring the populations for all settings are part of the same cohort. Three quarters of the community respondents indicated some limitations in the sensory attributes of hearing, vision, or speech, whereas the institutional population distribution indicates low functional ability in most attributes. Nearly all (97\%) of the NPHS community sample is independent in dexterity and using the algorithm used by Statistics Canada, they are classified as having no impairments in self-care. The high prevalence of independence in self-care for the NPHS is attributable to the use of HUI3 questionnaire items that assess hand and arm dexterity and the omission of the direct measurement of self-care.

Table 4 presents the results of the MDS-HSI scores for each setting, by age group and gender. On average, the institutional residents experience a lower mean score than the community dwelling population. This represents a lower measured health-related quality of life, a result that supports the discriminant validity of the measure. Individuals requiring care in a chronic hospital, nursing home, or residential facility would be expected to have, on average, a lower health status than the cohort residing in a community settings.

NPHS scores reported in Table 4 represent scores that were derived from items on the HUI3 survey instrument but use HUI2 preference weights (39). The general community NPHS population displays a higher HRQOL than the two MDS community cohorts.

Table 4. MDS-HSI (Mean \& Cl): Study Population and NPHS Comparisona

\begin{tabular}{|c|c|c|c|c|c|c|}
\hline \multirow[b]{2}{*}{$\begin{array}{l}\text { Age } \\
\text { category }\end{array}$} & \multicolumn{6}{|c|}{ Mean MDS-HSI (95\% CI) } \\
\hline & $\begin{array}{l}\text { Supportive } \\
\text { housing }\end{array}$ & Home care & $\begin{array}{l}\text { Resident } \\
\text { facility }\end{array}$ & $\begin{array}{l}\text { Chronic } \\
\text { hospital }\end{array}$ & $\begin{array}{c}\text { NPHS } \\
\text { community }\end{array}$ & $\begin{array}{c}\text { NPHS } \\
\text { institution }\end{array}$ \\
\hline Number & 80 & 353 & 165 & 274 & 3002 & 1467 \\
\hline \multicolumn{7}{|l|}{ Age } \\
\hline $65-69$ & $\begin{array}{c}0.92 \\
(0.80,1.04)\end{array}$ & $\begin{array}{c}0.58 \\
(0.54,0.62)\end{array}$ & $\begin{array}{c}0.36 \\
(0.32,0.40)\end{array}$ & $\begin{array}{c}0.38 \\
(0.32,0.44)\end{array}$ & $\begin{array}{c}0.81 \\
(0.80,0.83)\end{array}$ & $\begin{array}{c}0.58 \\
(0.54,0.63)\end{array}$ \\
\hline $70-74$ & $\begin{array}{c}0.79 \\
(0.65,0.93)\end{array}$ & $\begin{array}{c}0.55 \\
(0.49,0.61)\end{array}$ & $\begin{array}{c}0.31 \\
(0.13,0.49)\end{array}$ & $\begin{array}{c}0.36 \\
(0.28,0.44)\end{array}$ & $\begin{array}{c}0.79 \\
(0.78,0.81)\end{array}$ & $\begin{array}{c}0.58 \\
(0.54,0.62)\end{array}$ \\
\hline $75-79$ & $\begin{array}{c}0.80 \\
(0.68,0.92)\end{array}$ & $\begin{array}{c}0.59 \\
(0.53,0.65)\end{array}$ & $\begin{array}{c}0.31 \\
(0.17,0.45)\end{array}$ & $\begin{array}{c}0.36 \\
(0.30,0.42)\end{array}$ & $\begin{array}{c}0.77 \\
(0.75,0.79)\end{array}$ & $\begin{array}{c}0.56 \\
(0.52,0.59)\end{array}$ \\
\hline $80-84(80+)^{b}$ & $\begin{array}{c}0.82 \\
(0.76,0.88)\end{array}$ & $\begin{array}{c}0.58 \\
(0.54,0.62)\end{array}$ & $\begin{array}{c}0.33 \\
(0.23,0.43)\end{array}$ & $\begin{array}{c}0.33 \\
(0.27,0.39)\end{array}$ & $\begin{array}{c}0.72 \\
(0.70,0.74)\end{array}$ & $\begin{array}{c}0.52 \\
(0.50,0.55)\end{array}$ \\
\hline $85-89$ & $\begin{array}{c}0.77 \\
(0.71,0.83)\end{array}$ & $\begin{array}{c}0.54 \\
(0.50,0.58)\end{array}$ & $\begin{array}{c}0.29 \\
(0.19,0.39)\end{array}$ & $\begin{array}{c}0.27 \\
(0.21,0.33)\end{array}$ & $\mathrm{b}$ & $\begin{array}{c}0.52 \\
(0.50,0.54)\end{array}$ \\
\hline $90+$ & $\begin{array}{c}0.73 \\
(0.63,0.83)\end{array}$ & $\begin{array}{c}0.58 \\
(0.52,0.64)\end{array}$ & $\begin{array}{c}0.27 \\
(0.19,0.35)\end{array}$ & $\begin{array}{c}0.25 \\
(0.17,0.33)\end{array}$ & $\mathrm{b}$ & $\begin{array}{c}0.49 \\
(0.47,0.52)\end{array}$ \\
\hline \multicolumn{7}{|l|}{ Gender } \\
\hline Female & $\begin{array}{c}0.78 \\
(0.74,0.82)\end{array}$ & $\begin{array}{c}0.57 \\
(0.55,0.59)\end{array}$ & $\begin{array}{c}0.30 \\
(0.26,0.34)\end{array}$ & $\begin{array}{c}0.31 \\
(0.27,0.35)\end{array}$ & $\begin{array}{c}0.80 \\
(0.79,0.81)\end{array}$ & $\begin{array}{c}0.56 \\
(0.53,0.58)\end{array}$ \\
\hline Male & $\begin{array}{c}0.80 \\
(0.72,0.88)\end{array}$ & $\begin{array}{c}0.59 \\
(0.55,0.63)\end{array}$ & $\begin{array}{c}0.40 \\
(0.34,0.46)\end{array}$ & $\begin{array}{c}0.38 \\
(0.34,0.42)\end{array}$ & $\begin{array}{c}0.77 \\
(0.76,0.78)\end{array}$ & $\begin{array}{c}0.52 \\
(0.51,0.53)\end{array}$ \\
\hline
\end{tabular}

\footnotetext{
${ }^{a}$ Data scored as 0.0 (dead) to 1.0 (full health). MDS-HSI, Minimum Data Set Health Status Index; NPHS, National Population and Health Survey; CI, confidence interval.

${ }^{\mathrm{b}}$ Highest age category for NPHS community respondents represents all individuals $80+$ years old.
} 
Analogous to the study population, older NPHS cohorts generally experience a lower health status. Nonetheless, age appears to have less impact than health problems on index results (using residence setting as a proxy for health problems). The overall results for males are consistently higher than those of females, but those gender differences are significant only for the youngest cohorts when age is taken into account (data not shown).

MDS-HSI single-attribute scores were calculated using the HUI2 single-attribute weights (42). These weights are similar to multi-attribute scores shown in Table 2 but are independent of other attribute scores. For single-attribute scores, 0 (zero) represents the lowest possible functioning and 1 represents full functionality. The correlation between the MDS cognition (CPS) with the MDS-HSI single-attribute scores for cognition and between the ADL hierarchy scale and self-care and mobility attribute scores are used to evaluate the content and construct validity of these two MDS-HSI attributes. The intra-class correlation coefficient (ICC) between the CPS and the cognition attribute scores is 0.94 and the ICC for the MDS hierarchical ADL scale is 0.62 with the mobility attribute score and 0.57 with the self-care attribute score. (All reported correlation statistics reported here are significant using a $p$ value of 0.01.) Because the MDS-HSI cognition domain is based on the CPS, the high correlation is not surprising. The lower correlation for mobility and self-care is attributable to differences in the items for each scale. Only the mobility attribute includes the use of mechanical aids, only the self-care attribute includes bathing and dressing, and self-performance in hygiene is included only in the ADL hierarchy.

Single-attribute scores were also compared with summated scores of the MDS items associated with each attribute (scores for items associated with each attribute were added together). The ICC for the overall MDS-HSI score and a summated score of all items was 0.90 . The ICC between single-attribute and attribute-specific summated scores were emotion (0.89), mobility (0.83), cognition (0.92), self-care (0.92), pain (0.84), and sensation (0.78). The high level of correlation in all these comparisons suggests substantial agreement between the MDS- HSI attribute scores and summated scores of items associated with each health attribute.

The mean summated scores and MDS-HSI scores associated with each attribute, independent of other attributes, for each setting are displayed in Figure 1. Bars in this figure represent the mean single-attribute score on a continuous scale ranging from 0 (most impaired) to 1.0 (full functioning). The reported score is an average of individual scores calculated by using the preference weight associated with each resident's level of functioning. All attribute scores based on the MDS-HSI (for SH, HC, RF, and $\mathrm{CCH}$ settings) are lower for institutional residents than those for community based populations. These results are mirrored by the summated score results. Consistent with our hypotheses, mobility, selfcare, and cognition declines steadily with more intensive care settings. Supportive housing residents show higher health status than home care recipients in all domains. Residential facility residents show higher scores than chronic care hospital residents in mobility, self-care and pain outcomes, but slightly lower scores for sensation and emotion.

\section{Limitations}

This study has several important limitations. The small sample size limits the ability to examine low prevalence groups, and the number and range of care settings could be broadened. Comparisons across settings should be made with caution as there are only one or two organizations included in each population. Comparisons to the NPHS survey should also be made with caution. The NPHS community dwelling survey includes many elderly individuals who would have few health problems, whereas individuals in the supportive housing and home care study populations are known to have experienced some deterioration in health. Although the latter two groups receive support in the form of health and 


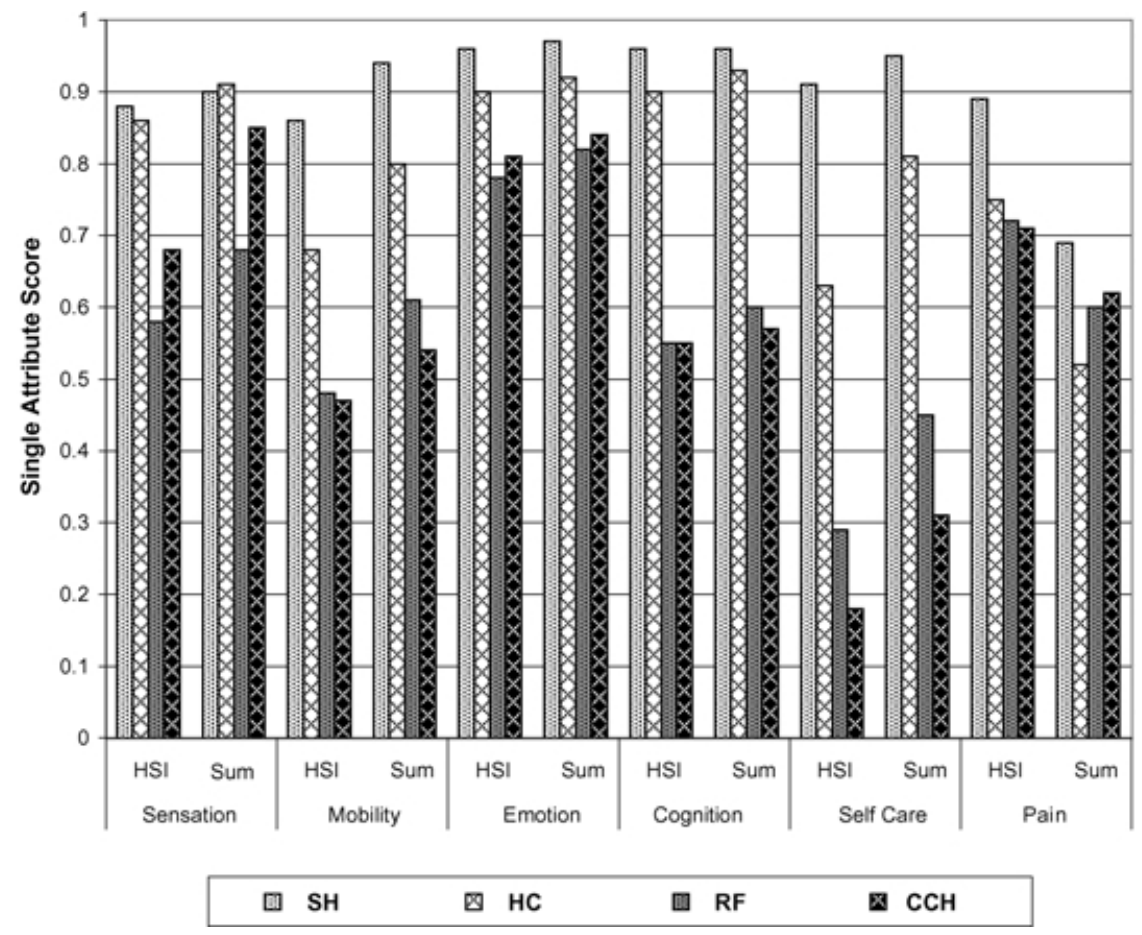

Figure 1. Single-Attribute Minimum Data Set Health Status Index and summated scores. HIS, Health Status Index; SH, supportive housing, HC, home care; RF, residential facility; $\mathrm{CCH}$, chronic care hospital.

personal care services, the data do indicate the extent and level of utilization of formal or informal care by NPHS community respondents.

Because MDS items are not identical to HUI2 definitions, a further assessment of the correspondence between MDS-HSI scores and HUI2 scores derived from the original HUI2 questionnaire is needed. More research is required to validate further the HUI2 for use with frail elderly populations. No assessment has yet been made to ensure that long-term care populations would give the same preference scores to the various health states summarized in the HUI2. The assessment of preference weights for well, frail, and ill elderly individuals is necessary to evaluate potential differences for these populations. Although the present results are valid for group level analyses, individual level analyses would require preference weights obtained from residents in the present study population. The ability of the MDS-HSI to assess change over time requires additional research. The responsiveness or sensitivity of the MDS-HSI to change is particularly important for outcome studies and clinical trials, and this should be examined in a prospective longitudinal study. The feasibility of such research is supported by our findings.

Comparing the results of the MDS mapping with the data obtained from the NPHS requires caution. The scoring of health status assessments from the NPHS was facilitated by means of a mapping to the HUI2 attribute definitions. Some of the indicators captured by the NPHS are not identical to the HUI2 measures as included in the MDS-based mapping algorithm. We cannot determine the comparability of NPHS community residents with home care and supportive housing residents because no information was available for NPHS community respondents' use of formal or informal care. The health institution data (NPHSHI) include residents of hospitals, nursing homes, and residential facilities for people with disabilities. Despite these caveats, the general trends depicted by the national data are useful. 


\section{Conclusions}

The results reported here provide preliminary support for the validity of the MDS-HSI as a suitable HRQOL measure for long-term care populations. The MDS-HSI supports stated hypotheses and appears useful in identifying the absolute and relative health status of individuals in each of the four long-term care settings.

The single-attribute MDS-HSI scores and summated scores provided qualitatively similar results and displayed a high correlation, hence providing support for construct and convergent validity. It is important to point out, however, that only the MDS-HSI scores can be used to directly compare magnitudes in different domains. This use is because the summated scores do not account for potential differences in the importance or weight that should be attributed to different levels of functioning nor do they provide a means for comparing the importance or weight that should be attributed to different levels of functioning for different health attributes.

The MDS-HSI scores were higher in the community settings than in the institutional settings, providing support for the discriminant validity of the measure. Problems with self-care, mobility, and cognition were all more apparent in the institutionalized elderly. Although not explicitly identified in our hypotheses, sensation was also more impaired in the institutional population. Emotion and pain could not be used to clearly distinguish between institutional and community dwelling populations.

\section{DISCUSSION}

HRQOL has gained popularity as the methodology for its measurement has become increasingly sophisticated and robust. Health researchers have carried out numerous studies to extract and define dimensions that contribute to HRQOL. The HUI2 is one existing measure for which there is extensive evidence of reliability and validity. The MDS-HSI summarizes the six attributes of HRQOL that are included in the HUI2. These attributes are particularly relevant for the elderly population. A single summary score does not fully capture subtle differences in individual experience, but it does provide a valid and convenient measure of the general outcomes of client care.

Although the MDS-HSI results provided support for our hypotheses, a few findings were not anticipated. Supportive housing residents score higher on overall and singleattribute scores than home care residents. This finding suggests that the former residents have higher functional status, despite dwelling in facilities that specifically offer personal care services. These results are reasonable, however, because not all supportive housing residents receive health care services. Additionally, there is potential for an unobserved income effect. Selection into supportive housing by wealthier residents who can afford to live in this setting may occur, whereas the community population may represent a broader spectrum of income. We can only speculate whether supportive housing residents are more stable and have better access to care that can prevent functional decline.

Although self-care impairment is more severe in the facility settings, some caution must be exercised in interpreting this result. It is often a legal requirement for institutional staff to assist residents in preparing for a bath, an item that is included in the self-care attribute definition (see Table 2). The notably higher levels of mobility impairment are not subject to the latter bias.

Ongoing evaluation efforts in long-term care would benefit from an overall HRQOL measure. Clinical trials have begun recently to incorporate aspects of health-related quality of life as outcome indicators. Both clinical trials and evaluations are used to inform decisions about care, interventions, new technologies, resource allocation, and health policy. These decisions need to be based on reliable and valid evidence. In comparative evaluations, the 
HRQOL measure can be used as a single summary score, representing several outcomes in a common form. Hence, HRQOL can help in determining whether interventions are truly effective.

An additional feature measures like the HUI2 is the ability to use the scores to integrate mortality and morbidity in quality-adjusted life-years (QALYs) or health-adjusted life expectancy (HALE). The MDS-HSI can be combined with life expectancy tables to provide the former measures. These types of measures, although still providing only cursory indicators of population health, are an improvement over the current standard of life expectancy alone.

\section{POLICY IMPLICATIONS}

The HRQOL indicator derived from MDS items provides several opportunities for use by health service providers, payers, clients, and researchers. Long-term care is expected to be among the most rapidly growing components of health care in North America and other parts of the world. Researchers have long argued for the implementation of a common approach to health status assessment across settings. The widespread use of the MDS offers an important opportunity to develop the capacity for national and international comparisons of patient health status and outcomes. The availability of adequate common information is important for care planning, case- mix funding, health status outcomes, quality improvement, and future system-wide planning. The MDS-HSI may provide a useful summary measure that goes beyond traditional indicators (e.g., mortality), for evaluating global outcomes of interventions in community and institutional settings. The primary benefit of linking this HRQOL measure to the MDS is that it will be available for large populations (due to mandated use) with no need for additional data collection.

It is increasingly important to assess the success of long-term care in maintaining or even improving health and quality of life. This assessment requires broad individual and system outcomes as part of the evaluation criteria. These must be largely independent of care setting characteristics. The MDS-HSI offers an opportunity to assess the status of clinical, facility, and system outcomes in long-term care. The algorithm and scaling weights for the MDS-HSI are available for use in research and evaluation purposes. Future experimentation with this instrument will help to improve the assessment of HRQOL in the frail elderly population.

\section{NOTE}

${ }^{1}$ The NPHS includes questions based on the HUI3 system. HUI3 consists of eight attributes: vision, hearing, speech, ambulation, dexterity, emotion, cognition, and pain. For Statistics Canada, Torrance et al. [43] mapped HUI3 responses into the HUI2 system to provide provisional preference scores, using an algorithm much like the one developed here for MDS assessment information. Derived health preference scores, obtained using data on health status from the 1994 NPHS for community $[\mathrm{n}=3,002]$ and institutional $[\mathrm{n}=1,467]$ residents sixty-five years of age and older, are used here as a comparison population. The overwhelming majority of responses for the NPHS community sample are based on self-assessment. Proxy respondents were often used in the NPHS institutional sample.

\section{REFERENCES}

1. Aller LJ, Van Ess Coeling H. Quality of life: Its meaning to the long-term care resident. J Gerontol Nurs. 1995;2:20-25.

2. Baladan DJ, Sagi, PC, Goldfarb NJ, Nettler S. Weights for scoring the Quality of Well Being Instrument among rheumatoid arthritics: A comparison to general public weights. Med Care. 1986;24:973-980.

3. Bergner M, Bobbitt RA, Carter WB, Gilson BS. The Sickness Impact Profile: Development and final revision of a health status measure. Med Care. 1981;19:787-805. 
4. Birren JE, Dieckmann L. Concepts and content of quality of life in the later years: An overview. In: Birren JE, Lubben JE, Rowe JC, Deutchman DE, eds. The concept and measurement of quality of life in the frail elderly. San Diego: Academic Press; 1991:344-360.

5. Berthelot JM, Roberge R, Wolfson MC. The calculation of health-adjusted life expectancy for a Canadian province using a multi-attribute utility function: A first attempt. Ottawa: Social and Economic Studies Division, Analytical Studies Branch, Statistics Canada; 1992.

6. Bond J. Quality of life for people with dementia: Approaches to the challenge of measurement. Ageing Soc. 1999;19:561-579.

7. Busschbach JJ, Hessing DJ, DeCharro FT. The utility of health at different stages of life: A quantitative approach. Soc Sci Med. 1993;37:153-158.

8. Crooks VC, Schnelle JF, Ouslander JP, McNees MP. Use of the minimum data set to rate incontinence severity. J Am Geriatr Soc. 1995;43:1363-1369.

9. Dolan, P, Gudex C, Kind P, Williams A. The time trade-off method: Results from a general population study. Health Econ. 1996;5:141-154.

10. Essink-Bot ML, Krabe PF, Bonsel GJ, Aaronson NK. An empirical comparison of four generic health status measures. The Nottingham Health Profile, the Medical Outcomes Study 36-item Short Form Health Survey, the COOP/WONCA charts, and the EuroQol Instrument. Med Care. 1997;35:522-537.

11. EuroQol Group. EuroQol: A new facility for the measurement of health-related quality of life. Health Policy. 1990;16:199-208.

12. Frederiksen K, Tariot P, De Jonghe E. Minimum Data Set Plus (MDS+) scores compared with scores from five rating scales. J Am Geriatr Soc. 1996;44:305-312.

13. Fries BE, Schneider DP, Foley JW, et al. Refining a case-mix measure for nursing homes: Resource Utilization Groups (RUG-III). Med Care. 1994;32:668-685.

14. Froberg DG, Kane RL. Methodology for measuring health-state preferences: Population and context effects. J Clin Epidemiol. 1989;42:585-592.

15. Furlong WJ. Variability of utility scores for health states among general population groups. MSc thesis, McMaster University; 1996.

16. Gold MR., Patrick DL, Torrance GW, et al. Identifying and valuing outcomes. In: Gold MR, Siegel JE, Russell LB, Weinstein MC, eds. Cost-effectiveness in health and medicine. New York: Oxford University Press; 1996:82-134.

17. Hadorn DD, Uebersax J. Large-scale outcome evaluation: How should quality of life be measured? I. Calibration of a brief questionnaire and a search for preference subgroups. J Clin Epidemiol. 1995;46:607-618.

18. Hartmaier SL, Sloane PD, Guess HA, Koch GC. Validation of the Minimum Data Set Cognitive Performance Scale: Agreement with the Mini-Mental State Examination. J Gerontol Med Sci. 1995;50A:M128-M133.

19. Hawes C, Morris JN, Phillips CD, et al.. Reliability estimates for the Minimum Data Set for nursing home resident assessment and care screening (MDS). Gerontologist. 1995;35:172-178.

20. Hays RD, Siu AL, Keeler E, et al. Long-term care residents' preferences for health states on the Quality of Well-Being Scale. Med Decis Making. 1996;16:254-261.

21. Hirdes JP, Zimmerman D, Hallman KG, Soucie PS. Use of the MDS quality indicators to assess quality of care in institutional settings. Can J Qual Health Care. 1998;14:5-11.

22. Hirdes JP, Carpenter GI. Health outcomes among the frail elderly in communities and institutions: Use of the minimum data set (MDS) to create effective linkages between research and policy. Can J Aging/Can Public Policy. (Special Joint Issue). 1997;(Suppl):53-69.

23. Hirdes JP, Fries BE, Morris JN, et al. Integrated health information systems based on the RAIMDS series of assessment instruments. Healthc Manage Forum. 1999;12:30-40.

24. Kaplan RM. Value judgment in the Oregon Medicaid experiment. Med Care. 1994;32:975-988.

25. Kaplan RM, Bush JW. Health-related quality of life measurement for evaluation research and policy analysis. Health Psychol. 1982;1:61-66.

26. Martin AJ, Glasziou PP, Simes RJ, Lumley T. A comparison of standard gamble, time trade-off, and adjusted time trade-off scores. Int J Technol Assess Health Care. 2000;16:137-147.

27. McDowell I, Newell C. Measuring health: A guide to rating scales and questionnaires. New York: Oxford University Press; 1996:36. 
Wodchis et al.

28. Morris JN, Fries BE, Steel K, et al. Comprehensive clinical assessment in community settings: Applicability of the MDS-HC. J Am Geriatr Soc. 1997;45:1017-1024.

29. Morris JN, Nonemaker S, Murphy K, et al. Commitment to change: Revision of HCFA's RAI. $J$ Am Geriatr Soc. 1997;45:1011-1016.

30. Morris JN, Fries BE, Morris SA. Scaling ADL's within the MDS. J Gerontol Med Sci. 1999;54:M546-M553.

31. Neumann PJ, Juntz KM, Leon J, et al. Health utilities in Alzheimer's disease: A cross-sectional study of patients and caregivers. Med Care. 1998;37:27-32.

32. Patrick DL, Deyo RA. Generic and disease-specific measures in assessing health status and quality of life. Med Care. 1989;3:S217-S232.

33. Patrick DL, Erickson P. Health status and health policy: Quality of life in health care evaluation and resource allocation. New York: Oxford University Press; 1993:22.

34. Patrick DL, Sittampalam Y, Somerville SM, Carter WB, Bergner M. A cross-cultural comparison of health status values. Am J Public Health. 1985;75:1402-1407.

35. Phillips CD, Morris JN. The potential for using administrative and clinical data to analyze outcomes for the cognitively impaired: An assessment of the Minimum Data Set for nursing homes. Alzheimer Dis Assoc Disord. 1998;11:162-167.

36. Phillips CD, Hawes C, Morris JN, Mor V, Fries BE. Effects of the RAI on quality of care: Executive summary. Research Triangle Park, NC: Research Triangle Institute, Program on Aging and Long-term Care; 1996.

37. Richardson J, Nord E. The importance of perspective in the measurement of quality ADJUSTED LIFE YEARS. Med Decis Making. 1997;17:33-41.

38. Rissanen P, Sogaard J, Sintonen H. Do QOL instruments agree? A comparison of the 15D and NHP in hip and knee replacements. Int J Technol Assess Health Care. 2000;16:696-705.

39. Roberge R, Berthelot J-M, Wolfson M. Health and socio-economic inequalities. Canadian social trends. Ottawa: Minister of Industry, Supply and Technology; 1995:15.

40. Sackett DL, Torrance GW. The utility of different health states as perceived by the general public. J Chron Dis. 1978;31:697-702.

41. Sgadari A, Morris JN, Fries BE, et al. Efforts to establish the reliability of the RAI. Age Ageing. 1997;26(Suppl 2):27-30.

42. Torrance GW, Feeny DH, Furlong WJ, et al. Multiattribute utility function for a comprehensive health status classification system (Health Utilities Index Mark 2). Med Care. 1996;34:702-722.

43. Torrance GW, Furlong W, Feeny D, Boyle MH. Provisional Health Status Index for the Ontario Health Survey. Hamilton: McMaster University; 1992.

44. United States Congress. Omnibus Budget Reconciliation Act OBRA. 1987. Public Law 100-203. Nursing Home Reform Act. 1819 (42 USC).

45. Ware JE, Sherbourne CD. The MOS 36-item Short-Form Health Survey (SF-36). I. Conceptual framework and item selection. Med Care. 1992;30:473-483.

46. Werko L. Health technology assessment at the end of life: A realistic view of death and palliative care. Int J Technol Assess Health Care. 2000;16:903-923.

47. Wieland D, Rubenstein LV, Hirsch SH. Quality of life in nursing homes: An emerging focus of research and practice. In: Katz PR, Kane, RL, eds. Quality care in geriatric settings: Focus on ethical issues. New York, NY: Springer; 1995:149-194.

48. Williams BC, Fries BE, Foley WJ, Schneider D, Gavazzi M. Activities of daily living and costs in nursing homes. Healthc Financ Rev. 1994;15:117-135. 